\title{
Design and development of a process-economic mathematical model of a lupine hydrolysis unit
}

\author{
Jiři Pecha ${ }^{1}$, Lubomir Š́nek ${ }^{1 *}$, Miloš Jelínek ${ }^{2}, J_{a k u b ~ H u s a ́ r}{ }^{1}$, and Karel Kolomazník ${ }^{1}$ \\ ${ }^{1}$ Tomas Bata University in Zlin, Faculty of Applied Informatics, Nad Stranemi 4511, 76005 Zlin, Czech Republic \\ ${ }^{2}$ University of Hradec Králové, Faculty of Science, Department of Informatics, Rokitanského 62, 500 03, Hradec Králové, Czech \\ Republic
}

\begin{abstract}
Lupine is considered as a source of protein with a high level of valuable amino acids, what can have in a human diet significant impact on the prevention of various diseases. This paper deals with the process modelling and computer simulation of the lupine flour hydrolysis, particularly focusing on calculations and comparisons of different process variants. Suggested process-economic mathematical model of the production process is a very effective tool for achieving optimal operating conditions under which the specific costs of preparing the final hydrolyzed product are minimal. Simultaneously, the proposed mathematical model of the production node of the hydrolysis unit includes the key processes for the preparation of the resulting hydrolyzate, i.e. the chemical reaction itself, filtration of the reaction mixture and concentration of the filtrate, which is the final desired product - the lupine hydrolysate.
\end{abstract}

\section{Introduction}

Proteins are important macronutrients with a source of essential amino acids and energy. Lupine or lupine flour is considered as a nontraditional source of proteins, soluble dietary fibre and, unlike cereals, lupine has a low content of starch. Lupine contains over $30 \%$ protein with a very high content of valuable essential amino acids, i.e. arginine, leucine, isoleucine, lysine, and simultaneously is classed among the gluten-free food [1, 2]. Proteins produced by the hydrolysis of these valuable sources may also elicit potent anticancer, antimicrobial, hypocholesterolemic, antihypertensive, antithrombotic, and antimicrobial effects [3, 4].

The basic process of hydrolysis technology of a solid substrate (e.g. defatted finely ground lupine flour) is the chemical reaction itself occurring in the reactor of the production unit. The final composition of the reaction mixture is defined by the selected reaction conditions, i.e. presence and type of catalyst, pressure, temperature, hydrodynamic conditions in a given process reactor, substrate concentration and reaction time. In our case, it is possible to use hydrolysis to remove starch from the input substrate or to get a protein hydrolyzate (in other words, to "extract" the protein fraction from the feedstock). The technological arrangement of both processes is similar; only the form of the final product differs.

The pattern of the production hydrolysis unit is shown in Figure 1.

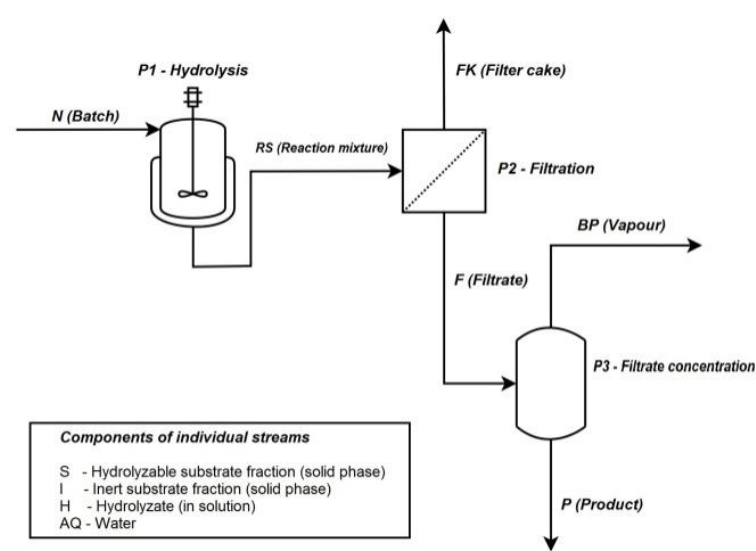

Fig. 1: Scheme of the production node of the hydrolysis unit including the list of considered components.

The figure presents three main processes of the technology, namely the chemical reaction itself hydrolysis of the solid substrate, separation of solids from the reaction mixture by filtration, and final concentration of the hydrolyzate (i.e. filtrate).

Based on the experimental measurements, the preparation of protein hydrolyzate, i.e. "extraction" hydrolysis, is a promising option. Therefore, the aim of this work is focused on the simulation calculations of this process variant. The process-economic mathematical model of the production process is a very useful tool for achieving this goal. But the mathematical model itself is also suitable for simulating and optimizing "refining"

* Corresponding author: sanek@utb.cz 
hydrolysis since the process layout is similar to that described above.

\section{Mathematical model of the production loop of the hydrolysis unit}

The input substrate itself is a very complex substance, but for the purposes of the mathematical model it is advisable to simplify its composition and only take into account the proportion of individual components involved in the reaction, the inert fraction, which is unchanged, the water, and the resulting product - i.e. the component that has reacted.

\subsection{Basic economic model}

In terms of the practical application of the mathematical model for the development and design of the industrial process, it is fundamental to involve economic parameters, as these determine the success and economic viability of the proposed technology. In this case, we will consider the cost of performing the chemical reaction and the cost of the resulting product concentrating to the desired level. Due to the process layout, the cost of filtration can be neglected. In particular, the cost of thickening the resulting product, i.e. the hydrolyzate, is expected to be determinative, but, as will be shown below, due to the complex rheological behavior of the reaction mixture, the cost of the implementation of reaction also occupies a significant portion of the total operating costs. Depreciation and wages are not included - they depend on the specific production capacity and production unit.

The cost for the realization of the chemical reaction is determined by the heat required to heat the reaction mixture and the electrical energy needed to homogenize the reaction mixture. The cost of maintaining the desired reaction temperature is negligible when the reactor is well insulated. However, the cost of homogenizing the reaction mixture depends on the dwell time in the reactor. The designed mathematical model works with a batch reactor, therefore it is also suitable for small-scale production. Let us add that due to the heterogeneity of the reaction system (solid substrate suspended in the liquid phase), the stirring intensity significantly increases the reaction rate and stirring of the batch further prevents the formation of any deposits, and for these reasons, we will only consider the variant of a stirred reactor.

From the discussion of the technology economy it follows that the main part of the operating costs $(N)$ of the hydrolyzate preparation consists of the cost of electricity $\left(N_{E}\right)$ needed to drive the stirrer of the hydrolysis reactor and the cost to achieve the desired concentration of hydrolyzate $\left(N_{O}\right)$, which is in practice achieved by evaporation of water from the filtrate (see Figure 1):

$$
N=N_{E}+N_{O}
$$

The costs of energy are determined by the product of the electromotor stirrer input power $P$, the reaction time $\tau$ and the unit price of electric energy $K_{E}$. The costs associated with the evaporation of water, it means the concentration of the product, are given by the multiplying of the heat of vaporization of water $\Delta H_{\mathrm{vyp}}$, the amount of evaporated water $m_{B D}$ and the unit price of thermal energy $K_{P}$. Therefore, the operating costs can be expressed as follows:

$N=K_{E} \cdot P \cdot \tau+K_{p} \cdot H_{v y p} \cdot m_{B D}$

The cost of electricity increases with increasing reaction time, but prolonging the reaction time leads to an increase in the product concentration in the reaction mixture, which reduces the price of the thickening. As a result, the major part of the operating expenses, depending on the reaction time, is minimal. The purpose of optimization is to find the minimum, i.e. the optimal reaction time at which the sum of costs is minimal. From a practical point of view, the specific costs are crucial. As the main product is thickened hydrolyzate, the following applies to the specific costs:

$N_{m}=\frac{N}{m_{P}}$

By optimizing the process according to specific costs, suitable operating conditions can be determined. The dependence of the total costs $N$ is more complicated (2), because the input power is a function of stirring intensity and reactor design, the total cost of the reaction is further influenced by the initial composition of the reaction mixture and other factors. For this reason, in the following chapters we describe in detail the mathematical model used to calculate total costs and discuss individual implemented functional dependencies.

\subsection{Kinetic model of the batch hydrolysis reactor}

It follows from the experimental data that the hydrolysis of the input substrate can be modeled with acceptable accuracy using first order kinetics. The mass balance of the hydrolyzable portion of the substrate (component "S" of process streams) in the batch reactor is the following:

$k \cdot a_{S, N} \cdot(1-x) \cdot m_{R S}=a_{S, N} \cdot m_{R S} \frac{d x}{d \tau}$

where $k$ denotes the hydrolysis rate constant valid for a given substrate, $x$ stands for the reaction conversion, $m$ is the stream weight and $a$ is the mass fraction. The subscripts denote the balanced component in accordance with the technological scheme (Figure 1).

Mass fractions instead of the usual concentrations were used in differential equation (4) for the reactant balance because this record takes into account both the experimental measurements and the kinetic data evaluation. The reaction time estimation depending on the desired conversion and vice versa are received by the integration of equation (4):

$\tau=-\frac{1}{k} \cdot \ln (1-x)$ 
$x=1-e^{-k \tau}$

From the mass balance it is possible to easily calculate the output composition of the reaction mixture, assuming that there is no evaporation of the volatile components of the reaction mixture:

$$
\begin{aligned}
& a_{H, R S}=a_{S, N} \cdot x \\
& a_{S, R S}=a_{S, N} \cdot(1-x) \\
& a_{A Q, R S}=a_{A Q, N} \\
& a_{I, R S}=a_{I, N}
\end{aligned}
$$

Due to the massive molar excess of water in the reaction phase it is justified to neglect water consumption for hydrolysis, as is clear from Eq. 9.

\subsection{Mathematical model of reactor batch homogenization}

The aim of this part of the mathematical modelling of the batch reactor is to estimate the input power of the electric motor needed to achieve the desired hydrodynamic mode of the reaction mixture. For the characterization of the hydrodynamic mode we used dimensionless Reynolds criterion $\left(R e_{M}\right)$, which takes the following form for stirring [5]:

$$
\operatorname{Re}_{\mathrm{M}}=\frac{n \cdot d^{2} \cdot \rho}{\eta}
$$

where $n$ stands for the stirrer rotational speed, $d$ is the stirrer diameter, $\rho$ stands for the reaction mixture density and $\eta$ denotes the reaction mixture dynamic viscosity.

The input power of the motor required to achieve the specified hydrodynamic mode can be calculated from the dimensionless input power criterion $P o$ [5], which is also often referred to as the Euler's Criterion [6]:

$$
\mathrm{Po}=\frac{P}{\rho \cdot n^{3} \cdot d^{5}}
$$

The dependence of $P o$ on $R e$ for various stirrer types can be found in the literature. From this dependency, it is evident that in a specific range of the Reynolds criterion of stirring, the value of the input power criterion is constant; this area is called automodel regime [6]. For mathematical modelling and simulation of the production process, we will practically in all cases work in the automodel regime and the input power criterion $P o$ will act as a constant in the mathematical model according to the type and construction of the stirrer. Due to the heterogeneity of the reaction system, it is desirable to work in the turbulent region, or at least in the transition region with the highest stirring intensity [7].

By modifying relations (11) and (12), we get the final relation for the calculation of the electromotor input power:

$P=\frac{\mathrm{Po} \cdot \mathrm{Re}_{\mathrm{M}}^{3} \cdot \eta^{3}}{\rho^{2} \cdot d}$

It can be seen from relation (12) that the input power significantly depends on the hydrodynamic conditions and the viscosity of the reaction system. It is also considerably influenced by the chosen stirrer and reactor design. For the possibility of variation of the reactor volume in the simulation calculations, constants are introduced into the mathematical model, the product of which with the reactor diameter gives the required geometric dimension, e.g. the level of the reaction mixture in the reactor or stirrer diameter $d$.

To calculate the input power of the electromotor, it is necessary to estimate the viscosity of the reaction system. Aqueous suspensions of solids containing a hydrolysable fraction (mainly starch) represent very complex systems in terms of quantitative description of their rheological behaviour [8-10]. A certain complication is a fact that these are usually nonNewtonian fluids $[8,9]$ and, besides, due to the ongoing hydrolysis, the viscosity changes during the reaction. The viscosity of the reaction system is, of course, further dependent on the concentration of the solid substrate in the suspension $[9,11]$.

For optimization and simulation of the influence of individual parameters on the modelled system, we made the following simplification - we considered the dependence of viscosity on the shear rate negligible for the reasons mentioned; therefore, we further assumed to be working with a Newtonian system.

To express the effect of the initial composition of the reaction mixture on its viscosity, we proceed from experimental data measured in [9] for rice flour suspension in water.

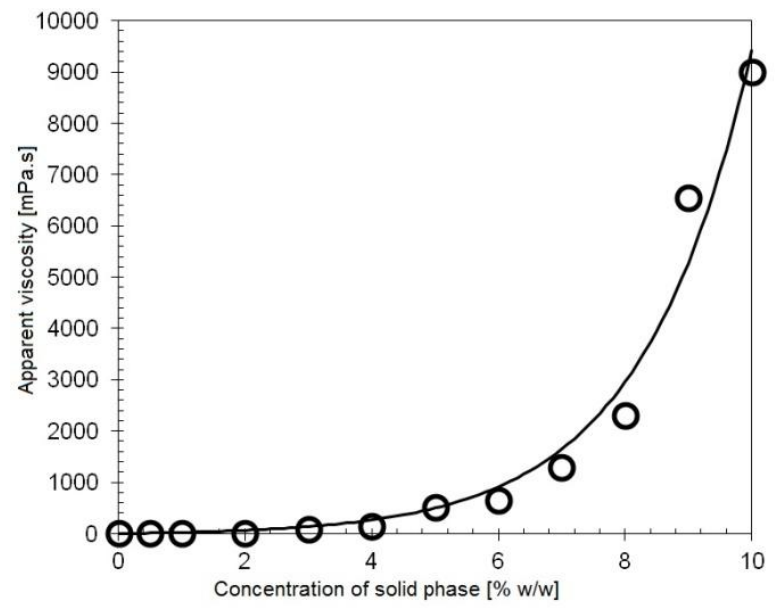

Fig: 2: Dependence of reaction mixture viscosity on the solid substrate concentration, circles indicate experimental data used from [9], the line indicates mathematical model data. 
Figure 2 shows the dependence of the reaction mixture viscosity on the concentration of the solid substrate and the fit of the experimental data by a mathematical model, which is further used to estimate the viscosity of the reaction system. This dependence is non-linear, i.e. increase in solid phase concentration results in a steep increase in viscosity.

\subsection{Mathematical model of subsequent processes of the technology}

As can be seen from the technological process diagram (Figure 1), the hydrolysis is followed by filtration of the reaction mixture and concentration of the filtrate. To calculate the total operating costs (1) (2), knowledge of the evaporated water amount is crucial. Furthermore, to calculate the specific operating costs, which are the most important from the practical point of view, it is necessary to quantify the amount of products produced - either the filter cake in the "refining" variant or the amount of filtrate in the "extraction" variant. All the above information can be obtained from the mass balance of both processes.

It is possible to estimate with a good accuracy from performed experimental work the output dry matter of the filter cake $\left(a_{D M},{ }_{F K}\right)$. It consists of an inert portion of feedstock which is not involved in the reaction and is still present in the solid phase and a part of the hydrolysable substrate which has not reacted under the reaction conditions. Filtrate, the dry matter of which contains the hydrolyzate, is not included in the dry matter of the filter cake. Thus, the filtrate retention is neglected. In practice, the filter cake is usually washed, so that the assumption mentioned above of the dry matter of the filter cake consisting only of the inert and unreacted portion is fulfilled. Accordingly, the mathematical model proposed below suggests transferring all of the hydrolyzate to the filtrate. From the mass balance of the process, including the above assumptions for the weight of the filter cake $\left(m_{F K}\right)$, follows:

$m_{F K}=\frac{m_{R S} \cdot\left[a_{S, N}(1-x)+a_{I, N}\right]}{a_{D M, F K}}$

The filtrate weight is then equal to:

$m_{F}=m_{R S} \cdot\left(1-\frac{\left[a_{S, N}(1-x)+a_{I, N}\right]}{a_{D M, F K}}\right)$

In addition to the weights of both streams, the concentration of hydrolyzate in the filtrate is essential. Based on the process mass balance, the following equation can be derived for the hydrolysate concentration:

$$
a_{H, F}=\frac{a_{S, N} \cdot x}{1-\frac{\left[a_{S, N}(1-x)+a_{I, N}\right]}{a_{D M, F K}}}
$$

By evaporating the water from the filtrate, the resulting product is obtained - thickened hydrolyzate. It depends on the dry matter concentration requirement in the final product. For the calculation of the final product weight after evaporation of the required amount of water, based on the mass balance of the filtrate thickening process, the following relation arises:

$$
m_{P}=\frac{m_{R S} \cdot a_{S, N} \cdot x}{a_{H, P}}
$$

The amount of evaporated water is equal to:

$$
m_{B P}=m_{R S} \cdot\left(1-\frac{\left[a_{S, N}(1-x)+a_{I, N}\right]}{a_{D M, F K}}\right) \cdot\left(1-\frac{a_{H, F}}{a_{H, P}}\right)
$$

\section{Simulation calculations}

After the implementation of the mathematical model in computational and simulation programs (e.g. Matlab, Scilab etc.) it is possible to perform extensive simulation calculations. As was discussed in the previous chapters, it can be expected that the overall process costs have a minimum in a particular area. With total specific costs, the situation is somewhat more complicated because not only less water evaporates when high conversions are achieved, but more product is produced, which reduces overall specific costs. For this reason, the optimal conditions from the total and specific costs point of view may vary quite significantly. From a practical point of view, the specific costs are crucial, i.e. costs related to the amount of product per unit.

The simulation performed with the proposed mathematical model under the conditions summarized in Table 2 shows, that specific costs optimization can significantly improve the economy of the entire production process. Reynolds stirring criterion $R e_{M}$ was set to 5000. The calculated dependence of the main specific cost on conversion is shown in the figure (Figure 3). Under the given input parameters, it is clear that the optimal conversion is approximately $91 \%$. Note that the reaction rate used in the simulation was evaluated from experimental data and refers to "extraction" hydrolysis of lupine flour.

Of course, in addition to conversion, several other parameters significantly affect the overall production costs. As the discussion suggests in deriving of the mathematical model, sufficient stirring intensity is necessary to provide the required mass transport rate in the heterogeneous reaction system. The costs for batch stirring are significantly dependent on the hydrodynamic mode of the plant. 
Table 2: Parameter values used for simulation calculation.

\begin{tabular}{|c|c|c|}
\hline Parameter & Value & Unit \\
\hline$K_{E}$ & 6 & {$[\mathrm{Kč} / \mathrm{kWh}]$} \\
\hline$K_{P}$ & 600 & {$[\mathrm{Kč} / \mathrm{GJ}]$} \\
\hline$k$ & 0,034 & {$\left[\mathrm{~min}^{-1}\right]$} \\
\hline$a_{S, N}$ & 2,5 & {$[\% \mathrm{w} / \mathrm{w}]$} \\
\hline$a_{I, N}$ & 4,5 & {$[\% \mathrm{w} / \mathrm{w}]$} \\
\hline$\tau_{\text {ind }}$ & 60 & {$[\mathrm{~min}]$} \\
\hline$V_{R}$ & 5 & {$\left[\mathrm{~m}^{3}\right]$} \\
\hline$c h r$ & 1,4 & [1] \\
\hline $\mathrm{cmr}$ & 0,4 & [1] \\
\hline Po & 0,35 & [1] \\
\hline$\rho$ & 1000 & {$\left[\mathrm{~kg} \cdot \mathrm{m}^{3}\right]$} \\
\hline$a_{D M, F K}$ & 30 & {$[\% \mathrm{w} / \mathrm{w}]$} \\
\hline$a_{H, P}$ & 95 & {$[\% \mathrm{w} / \mathrm{w}]$} \\
\hline$\Delta H_{v y p}$ & $2,26 \cdot 10^{6}$ & {$\left[\mathrm{~J} \cdot \mathrm{kg}^{-1}\right]$} \\
\hline
\end{tabular}

Typ of stirrer propeller

$\overline{\tau_{\text {ind }}-\text { induction period, } V_{R}-\text { reactor volume, } c h r-\text { level reactor }}$ constant, $\mathrm{cmr}$ - stirrer diameter constant, $\rho$ - density of reaction mixture, $K_{E \text { - }}$ the price of electricity, $K_{P^{-}}$the price of steam

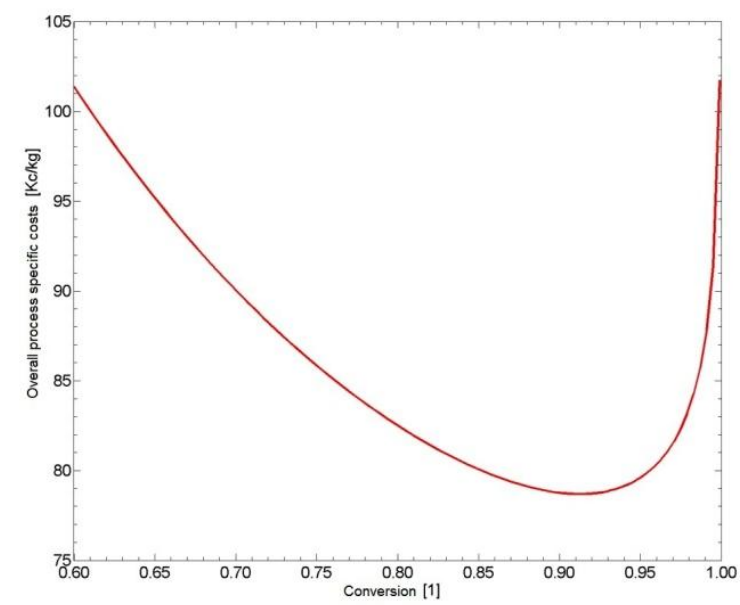

Fig. 3: Dependence of the main operating costs on conversion.

The mathematical model allows comparing the operating costs for hydrolyzate production at different stirring intensities expressed by the Reynolds criterion of stirring. The three-dimensional graph (Figure 4) shows the results of the simulation calculations; the values of the input data are summarized in Table 2. It can be seen from the simulation that the optimum conversion at which the lowest specific costs are achieved is dependent on the desired mixing intensity. It is advisable to reach higher conversions with decreasing mixing intensity. On the other hand, the specific reactor performance (amount of product manufactured during unit of time) is decreasing, which has to be compensated by reactor larger volume to ensure a constant production rate; thus the investment costs for the acquisition of the hydrolysis unit are increasing. Hence, the mathematical model enables comparison and optimization of the various operating modes of the industrial equipment.

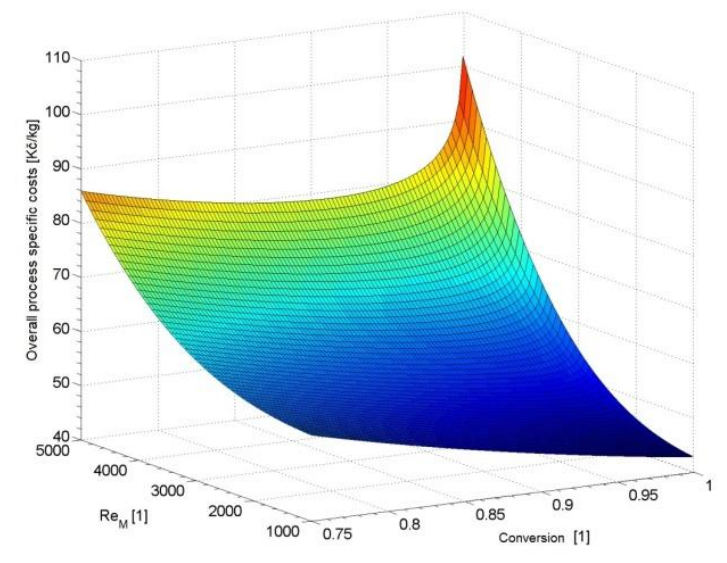

Fig. 4: Dependence of overall specific costs on conversion and $R e_{M}$.

From the viscosity measurements follows that this quantity significantly depends on the concentration of the solid input substrate in the reaction mixture. From the economic point of view, it is desirable to process as much concentrated suspension of a substrate in water as is possible because the specific performance of the hydrolysis unit is increasing while decreasing the cost of thickening the product. Hydrodynamic conditions influence the process rate, so it is desirable to keep these at a sufficiently high level. For this reason, we performed simulation calculations to describe the dependence of total specific conversion costs and initial input substrate. The observed dependence is presented in Figure 5, the table of values of the input parameters is summarized (Table 2), Reynolds stirring criterion $R_{M}$ was set to 2000 and mass fraction $a_{S, N}$ was calculated from relation $0,35 \cdot\left(a_{S, N}+a_{I, N}\right)$ specifiing initial composition of solid reactant - lupine flour.

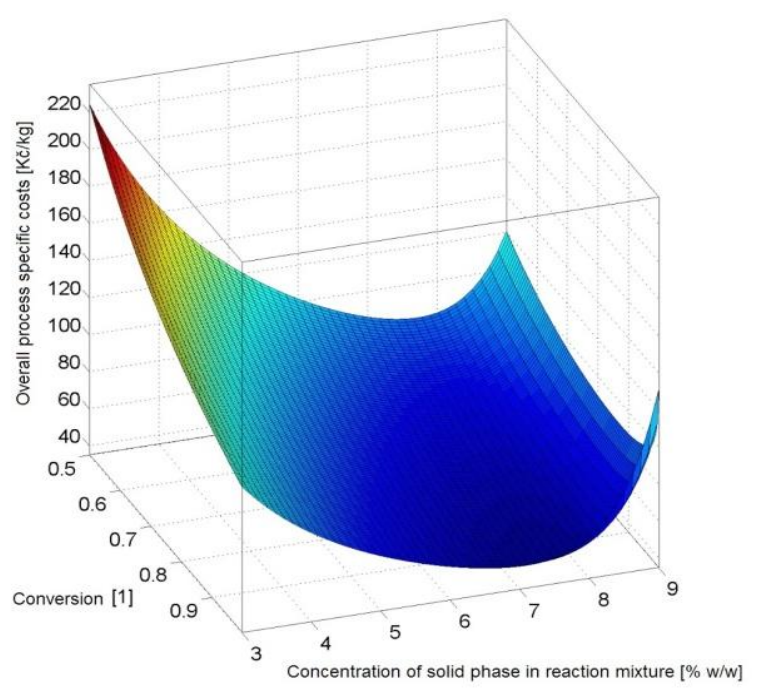

Fig. 5: Dependence of overall specific costs on conversion and initial solid phase concentration in the reaction mixture.

The results show that there is an economic minimum cost for a given conversion that corresponds to the specific value of the solid phase concentration of the feedstock in the reaction mixture. Similar dependencies 
can be compiled for a wide range working modes of the operating unit, allowing setting optimum conditions to ensure hydrolyzate economical production.

\section{Conclusion}

The proposed mathematical model of the hydrolysis unit includes the basic processes for the preparation of the resulting hydrolyzate, i.e. the chemical reaction itself, filtration of the reaction mixture and concentration of the filtrate, which is the final product. The mathematical model can be used for description of multiple technology variants, both for the "refining" technology and for "extraction" hydrolysis for which most simulation calculations have been performed.

The proposed mathematical model of the batch hydrolytic reactor, which forms the main part of the overall model, takes into account the reaction kinetics, the basic rheological parameters of the batch and the construction of the apparatus. As a result, it is possible to economically optimize the technology not only to find optimal operating conditions under which the specific costs of preparing the final product are minimal but also to assess their technological and technical availability and practical feasibility. The modelling has shown that in most of tested cases the economic optimum exists and thus the application of the model is valuable for the practical purposes.

This work was supported by the Ministry of Education, Youth and Sports of the Czech Republic within the National Sustainability Programme project No. LO1303 (MSMT- 7778/2014), by the European Regional Development Fund under the project CEBIA-Tech No. CZ.1.05/2.1.00/03.0089 and by the research project of Technology Agency of the Czech Republic (TACR), No. TA01010737 and also by the internal project No. RVO/CEBIA/2019/003.

\section{References}

1. S. Hubalovsky, J. Jelinek, M. Jelinek, K. Kolomazník, Conference; 5th, Recent researches in chemistry, biology, environment and culture, 132137 (2011).

2. K. Sedláková, E. Straková, P. Suchý, J. Krejcarová, I. Herzig, Acta Veterinaria Brno, 85, (2016).

3. M. d. C. Millán-Linares, B. Bermúdez, M. d. M. Yust, F. Millán, J. Pedroche, J. Funct. Foods, 8, (2014).

4. M. Chalamaiah, W. Yu, J. Wu, Food Chemistry, 245, (2018).

5. D. W. Green, R. H. Perry, Perry's Chemical Engineers' Handbook. 7th ed., New York: McGrawHill (1998).

6. J. Hanika, Vícefázové reaktory. Vyd. 1. Praha: Vysoká škola chemicko-technologická (1997).

7. J. Horák, J. Pašek, Návrh průmyslových chemických reaktorů z laboratornich dat. 1. vyd.
Praha: Státni nakladatelství technické literatury (1980).

8. E. B. Bagley, D. D. Christianson, J. Texture Stud., 13, (1982).

9. K. Prasad, A. Anil, Y. Yadav, A. Sinha, International Food Research Journal, 20, (2013).

10. H. Akdogan, J. Food Sci. Technol., 34, (1999).

11. A. Andrés, G. Acosta-Osorio, Herrera-Ruiz, F. Martínez-Bustos, , et al., Mech. Eng. Res., 1, (2011). 